\title{
Las ciencias sociales frente a las problemáticas medio-ambientales: apuntes de un primer encuentro
}

Lima, 12 de noviembre de 2014

Jérémy Robert y Karina Castañeda

\section{(2) OpenEdition}

Journals

Edición electrónica

URL: https://journals.openedition.org/bifea/6021

DOI: 10.4000/bifea.6021

ISSN: 2076-5827

Editor

Institut Français d'Études Andines

Edición impresa

Fecha de publicación: 1 diciembre 2014

Paginación: 668-674

ISSN: 0303-7495

\section{Referencia electrónica}

Jérémy Robert y Karina Castañeda, «Las ciencias sociales frente a las problemáticas medioambientales: apuntes de un primer encuentro», Bulletin de l'Institut français d'études andines [En línea], 43 (3) | 2014, Publicado el 08 diciembre 2014, consultado el 21 septiembre 2021. URL: http:// journals.openedition.org/bifea/6021 ; DOI: https://doi.org/10.4000/bifea.6021

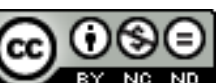

Les contenus du Bulletin de l'Institut français d'études andines sont mis à disposition selon les termes de la licence Creative Commons Attribution - Pas d'Utilisation Commerciale - Pas de Modification 4.0 International. 


\section{LAS CIENCIAS SOCIALES FRENTE A LAS PROBLEMÁTICAS MEDIO- AMBIENTALES: APUNTES DE UN PRIMER ENCUENTRO}

Lima, 12 de noviembre de 2014

En el contexto de la COP 20 que se realizará en Lima del $1^{\circ}$ al 12 de diciembre 2014, se han multiplicado los espacios de discusión sobre los temas ambientales: coloquios, seminarios, reuniones de trabajos, etc., que han logrado reunir a miembros de la sociedad civil, instituciones públicas, universidades, sector privado y ONG. Frente a la diversidad de los contenidos y objetivos, surgió la necesidad de aclarar la posición de la academia alrededor de dos preocupaciones: ¿Cuáles son las temáticas de investigación de las ciencias sociales frente a la problemática ambiental? ¿Cuáles son los elementos claves a incluir en una agenda de investigación a construir?

El INTE- PUCP viene impulsando en esta coyuntura las coordinaciones académicas e interuniversitarias así como el fortalecimiento de los grupos de trabajo. El punto de origen es la «Declaración de los académicos del Perú. Frente a los desafíos del cambio climático y a propósito de la vigésima conferencia de las partes», resultado de la tercera reunión de investigadores: «KAWSAYPACHA: Diálogos sobre la tierra» realizado en Lima en septiembre último.

Las cuestiones ambientales son también centrales en las investigaciones del IFEA ya que contribuyen a alimentar el debate y fortalecer las colaboraciones a nivel regional con miras a la COP 21 que se realizará en París. Como antecedente está la organización con la UMI IGlobes (Universidad de Arizona/CNRS) y el Cemca (Centro de Estudios Mexicanos y Centroamericanos) de las quintas Jornadas de Jóvenes Americanistas (JJA) «Nuevos acercamientos de los desafíos ambientales: las ciencias sociales frente a la adaptación a cambios climáticos. Perspectivas 
transdisciplinarias, formaciones y diálogos metodológicos», realizado en Tijuana, México en julio de este año y que se ha convertido en un antecedente clave (Robert, 2014). En esta coyuntura, el contexto peruano se prestaba entonces para la organización de un encuentro de esta naturaleza.

Continuando con las actividades de ambas instituciones, el Instituto Francés de Estudios Andinos (IFEA) y el Instituto de Ciencias de la Naturaleza, Territorio y Energía Renovable (INTE-PUCP) coincidieron con esta idea y decidieron impulsar una primera discusión realizada en la Pontificia Universidad Católica del Perú (PUCP) el 12 de noviembre del presente año bajo el título «Las ciencias sociales frente a las problemáticas medioambientales».

Esta actividad se propuso como un espacio de discusión académico desde las ciencias sociales sobre temas ambientales. El objetivo fue plantear líneas generales para contribuir a la construcción de una agenda de investigación y discutir las posibilidades de colaboración académica entre Francia y Perú.

El objetivo fue proponer una reflexión original sobre el papel de las ciencias sociales en las temáticas ambientales, enfocado en sus dimensiones sociales y políticas y en su reconfiguración contemporánea. ¿Cómo se analizan las políticas actuales o las dimensiones sociales y políticas de los problemas ambientales? ¿Cuáles son los marcos teóricos y conceptuales? ¿Cuáles son los desafíos y los grandes debates? Las dimensiones sociales y políticas son centrales y transversales a las problemáticas ambientales (pero obviadas a menudo), y permiten involucrar diferentes disciplinas (Ciencias Políticas, Derecho, Sociología, Antropología, Geografía, Economía, etc.). Como línea directriz, se propuso discutir del aporte de cada disciplina (líneas históricas de trabajo, evolución, desafíos actuales y reconfiguración de las temáticas) y de buscar ejes de investigación común.

El formato escogido para este evento fue un seminario/workshop de un día con un grupo limitado de participantes (25) de diferentes universidades e institutos de investigación para favorecer el intercambio de opiniones. Las discusiones se organizaron en 4 momentos: un primer panel introductorio que permitió plantear los elementos de contexto y las bases del debate con conferencias del Dr. Mathieu Durand (Universidad de Maine - IFEA, Francia) y del Dr. Augusto Castro (Director del INTE). Tres paneles temáticos permitieron los intercambios y las discusiones a partir de tres intervenciones cortas alrededor de la gestión del agua, de los conflictos ambientales, y de la economía y del derecho del medio ambiente.

Subrayaremos algunos de los temas debatidos de forma transversal durante las presentaciones y discusiones, en los paneles específicos. Estos apuntes no tienen la pretensión de presentar de forma exhaustiva y con total objetividad lo discutido en el seminario, sino más bien, resaltar lo que consideramos relevante considerar. 


\section{DEL ROL DE LA CIENCIA SOCIAL A LA PRODUCCIÓN DE CONOCIMIENTO}

\section{1. El rol de las ciencias sociales}

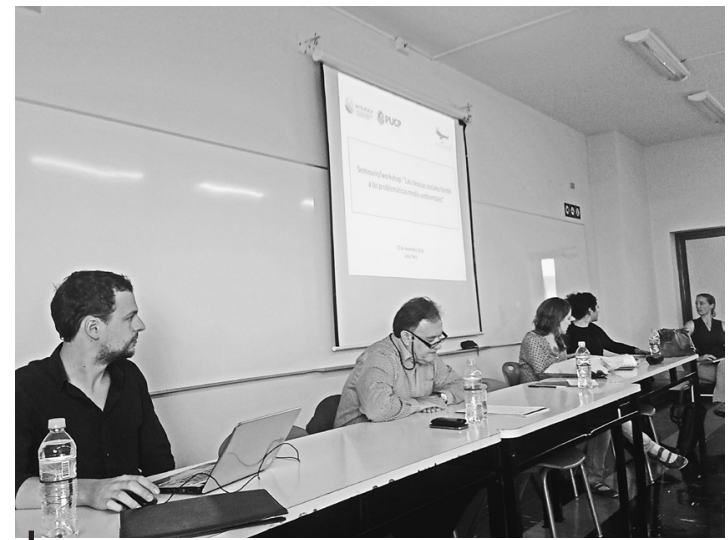

Desarrollo de una sesión de trabajo

Mathieu Durand inauguró las discusiones recordando cómo las ciencias sociales, y en particular la Geografía, habían acompañado los grandes paradigmas de las relaciones entre el hombre y la naturaleza y cómo habían contribuido a su construcción. Inicialmente enfocada en los problemas ambientales, las contaminaciones y el higienismo, se promueve en el siglo XIX una visión tecnicista segura de la abundancia de los recursos naturales y de las posibilidades de control gracias a los progresos tecnológicos. El panorama cambia radicalmente en las últimas décadas, donde surge un interés súbito por el tema ambiental, consciente de los límites del progreso (y de sus riesgos). Se trata hoy de buscar soluciones a los problemas del ambiente generados por el hombre. Se impone una necesidad política y práctica ¿Cómo materializar el «desarrollo sostenible»? En este contexto, se plantea el rol y la posición de las ciencias sociales, tanto como ingeniería social con un enfoque operacional, como crítica, para contribuir a nuevos conocimientos sobre los procesos en curso y los desafíos contemporáneos.

Frente a estos nuevos desafíos, Augusto Castro llamó a la necesidad de una visión reflexiva sobre la ciencia. Los problemas ambientales, por su dimensión antrópica, cuestionan la relación entre el objeto y el sujeto y la forma de hacer investigación. También la relación entre tecnología y cultura que son parte de una misma construcción social del mundo, y en la cual participan las ciencias sociales. Se llamó en este sentido al desarrollo de un conocimiento más global, sin dejar de reconocer la pluralidad de saberes y de visión del mundo. Los indígenas se identifican con su territorio en función de los ríos, el cambio climático se enfrenta a las prácticas ancestrales en las faldas del Huaytapallana (Téofilo Altamirano, PUCP). ¿Cómo los indígenas se apropian el derecho nacional e internacional para luchar contra las petroleras? ¿Cómo un comunero andino maneja al mismo tiempo las apus en quechua y las conclusiones del IPCC en inglés? Los saberes ancestrales se confrontan, se mezclan y se transforman con nuevos contextos. Somos espectadores $\mathrm{Y}$ productores de estas interacciones que generan nuevas formas de ver el mundo. Es parte del rol de las ciencias sociales restituir este conocimiento en su diversidad. 
Évènements

\section{2. Los medios de producción de conocimiento}

Nicolas Merveille (Universidad Antonio Ruiz de Montoya) cuestionó las condiciones de producción del conocimiento: ¿Quién maneja la información? ¿Esta se encuentra disponible? ¿Quién financia qué, y con qué fin? Mientras el sector minero y petrolero financia los estudios de impactos ambientales, poco medios se dedican al conocimiento del mundo. En este contexto, las ciencias sociales, contratadas por el sector interesado, restringen sus campos de análisis y sus críticas para entrar en un sistema de instrumentalización de su producción. Para evitar esto, defiende el desarrollo de una investigación autónoma y el libre acceso. Se planteó la importancia de una investigación reflexiva y crítica sobre su instrumentalización, capaz de distanciarse de una visión «clorofilizada» de la naturaleza, y provocar debates y evidenciar la construcción social de los problemas como la escasez o la abundancia de agua por ejemplo, tomando como referencia el trabajo del grupo GEAS1.

\section{DEL AGUA AL OBJETO DE INVESTIGACIÓN: DISCUSIÓN SOBRE LAS DISCIPLINAS Y LA FORMACIÓN}

\section{1. El agua}

El agua fue un tema central de las discusiones; motivo directo de 5 ponencias y abordada en otras tantas, parece ser el tema más emblemático de la problemática ambiental. La diversidad de los enfoques demuestra la riqueza de las ciencias sociales y la importancia del objeto de investigación. Teresa Oré (PUCP) comentó el camino de la construcción de un canal de riego en Ica a partir de una leyenda hacia los desafíos contemporáneos de la «gestión integrada de los recursos hídricos», viendo los cambios de los actores. Este camino ha sido también el suyo como investigadora, desde la antropo-historia hacia la sociología de las instituciones y las ciencias políticas al toparse con la «burocracia hídrica». La intervención de Patricia Urteaga (PUCP) parte del análisis del pluralismo legal en pueblos indígenas con una visión del derecho desde arriba, para buscar nuevos esquemas de análisis en la ecología política. Muestra la necesidad de enfocarse en los puntos ciegos generados por la segmentación de los problemas por las disciplinas.

La mirada del derecho sobre los recursos hídricos no se restringe a la definición oficial de su estatus, sino también a la regulación (Armando Guevara, PUCP). Existen formas alternativas al derecho oficial que dictan los Estados, y se pueden analizar como diferentes formas de regulación entre grupos sociales y en relación con los recursos naturales. Esta perspectiva evidencia dilemas y contradicciones,

1 Grupo de Estudios Ambiente y Sociedad de la PUCP, en particular su última publicación Oré \& Damonte, 2014. 
entre el derecho oficial — todas las fuentes de agua son del Estado- y la realidad social —las comunidades campesinas se consideran como dueñas del agua-u otros derechos — el agua como bien común-. En este contexto, se subrayó la importancia de contrastar la enunciación teórica con la materialización social. Pasa por la capacidad de cambiar de escala de observación: no limitarse el derecho internacional o el «telescopio del Estado», y tomar un «microscopio para entender la base». No limitarse tampoco a la foto que utiliza el legislador pero sí insertarse en una perspectiva histórica para entender los procesos en juego.

Eduardo Zegarra (Grade, Universidad Nacional Agraria La Molina) recordó la dificultad de los economistas al abordar la cuestión del agua, un bien que «no se puede cercar» y que no tiene un valor dado (más bien diferentes valores de naturaleza distinta). Así lo muestran Teófilo Altamirano (PUCP) y Jérémy Robert (IFEA, PUCP) con los conflictos alrededor del agua en Huancayo al pie del Huaytapallana, o en la cercanía de Lima en el valle de Santa Eulalia. En el primer caso, las prácticas ancestrales y costumbres, la urbanización creciente y los cambios ambientales generan tensiones para el acceso y la protección de un recurso que parece ser cada vez más escaso. En el segundo caso, se considera cómo los cambios climáticos van reconfigurando las movilizaciones sociales sobre la gestión del agua en Lima en el marco del proyecto Bluegrass². La ausencia de grandes conflictos sociales sobre el agua en Lima sorprende a primera vista, sin embargo se evidencian situaciones de tensiones, ya sea por privatización, por las tarifas del agua en Lima, o por el uso del agua entre diferentes sectores en el valle próximo de Santa Eulalia. El cambio climático parece redistribuir las cartas, dando oportunidades a nuevos grupos de incidir en las políticas públicas.

\section{2. Disciplinas y enfoques}

La Geografía ha sido presentada como una disciplina central, por su historia y sus especificidades para trabajar las relaciones entre hombre y medio ambiente, pero siempre recordando la necesidad de la pluridisciplinariedad. Si bien la Geografía presenta ciertas ventajas para moverse entre temas más «físicos» y otros más «sociales» (o más bien entre diferentes formas de abordar las cosas), presenta también el riesgo de un determinismo ambiental. Los aportes de otras ciencias sociales como la Sociología, el Derecho o la Antropología sobre el tema del agua (por ejemplo) han demostrado la fuerza (y la necesidad) de enfoques pluridisciplinarios.

Por su lado, la Economía se auto-ubica con cierta distancia de las otras ciencias sociales, por su propia historia y por la forma como ha venido abordando los

2 El proyecto BLUEGRASS es un programa de investigación ANR 2014-2016 coordinado por la UMI iGlobes (CNRS/Universidad de Arizona), en colaboración con el Cemca (México, UMIFRE 16, CNRS/ MAEDI), el Cirad (Centre international de recherche sur l'agriculture et le développement, Francia), el IFEA (Perú, UMIFRE 17, CNRS/MAEDI) y la Universidad de São Paulo (Brasil). Información disponible en: http://www.cemca.org.mx/noticias.php?id=1728\#.U9lyi-N5NJk 
problemas, a menudo con fórmulas matemáticas, un enfoque centrado en el individuo descontextualizado y la idea del rational choice (Eduardo Zegarra, Grade, Universidad Nacional Agraria La Molina; Manuel Ego, Universidad Antonio Ruiz de Montoya). Conscientes de los límites de este enfoque, muchos buscan caminos alternativos, reforzando lazos con otras ciencias sociales. La necesidad de ampliar el conocimiento es grande, en particular «cuando es la economía la que rige un país y orienta sus políticas» pero con poco análisis de los impactos. La problemática ambiental plantea nuevos retos y nuevas preguntas, acelerando el fin del utilitarismo y el reconocimiento de los límites del planeta y de las soluciones tecnológicas (Sofía Castro, INTE - PUCP). En este contexto, ¿Cómo pasar de la economía al compromiso político? ¿Cómo buscar y garantizar más equidad? ¿Por qué es importante priorizar la construcción de un metro en Lima (6 mil millones de USD) y no el agua potable en la selva? Coincidiendo con las otras disciplinas (o mejor dicho enfoques), se plantea la necesidad de un análisis crítico para descifrar estas «soluciones políticas disfrazadas de soluciones técnicas que cumplen con el SNIP».

Geografía, Economía, Ciencias Políticas, Sociología, Antropología, Derecho o Historia: los debates ha demostrado el aporte y la riqueza de enfoques desde otras disciplinas, llamando a un diálogo entre las ciencias sociales para compartir métodos, conceptos, y sobre todo, objetos de investigación. Las experiencias han mostrado que el camino de un investigador no es en línea recta. Gérard Borras subraya que la metodología (o la disciplina) no debe definir el objeto, pero más bien es el objeto que implica buscar y construir su propia metodología.

\section{3. ¿Especialización o generalización del conocimiento?}

Los temas ambientales plantean problemas globales donde el investigador se enfrenta tanto a problemas técnicos como a una diversidad de temas sociales, desde lo más específico hasta lo más general. Teresa Oré, desde su perspectiva de docente e investigadora sobre el tema del agua, se refirió a las «dos caras de una moneda», contando las diferencias de conocimientos y enfoque entre, de un lado los ingenieros hidráulicos o agrarios, y del otro lado los sociólogos. Coincidimos con la idea de que, «nadie puede ser especialista del agua», porque existe diversidad disciplinaria para abordar el tema. En este sentido, se subrayó la necesidad de combinar los conocimientos, cuestionando la capacidad de dialogar entre distintas áreas de investigación, ciencias sociales, y entre ciencias sociales y ciencias «duras».

En este contexto, la formación universitaria ha sido un tema recurrente de discusión. Se criticó la sobre especialización que puede llevar a la segmentación del conocimiento (por ejemplo, dividiendo el derecho de la tierra por un lado, del derecho del agua por otro). Estas brechas se evidencian con la sectorización de la gestión del Estado, subrayando las interrelaciones entre la academia y el mundo operacional y político. Una de las opciones es elevar la generalidad de la formación. Se defendió también la importancia de las especialidades. De la 
misma forma que las investigaciones empíricas son necesarias para llegar a alguna forma de generalización, la complejidad de los desafíos contemporáneos necesita especialistas, pero especialistas con capacidad de dialogar con otros especialistas, con el objetivo común de construir instrumentos de conocimiento. El debate sigue abierto.

¿Qué dicen las ciencias sociales sobre los problemas ambientales? El seminario mostró la diversidad de los enfoques y sus aportes para entender retos contemporáneos. El surgimiento de la temática ambiental en la agenda internacional, tanto mediática como política, plantea un nuevo contexto de trabajo. De un lado, nace un nuevo eje impulsado por las ciencias «duras» que buscan definir los límites del planeta y los escenarios de cambio y que piden a las ciencias sociales y a las sociedades, capacidad de adaptación. De otro lado, se mantiene una investigación en ciencia social más distanciada, fuerte en su historia, que cuestiona la construcción social de las categorías y de los conocimientos, recordando por ejemplo que la adaptación al cambio climático no debe ocultar la cuestión de las desigualdades sociales. En todo caso, se apunta a la construcción de una visión más integral de un mundo cada vez más complejo y más globalizado, regido por un conjunto de reglas (plurales) sociales, económicas, políticas y legales. Con este fin, se cree en la importancia del debate y del reconocimiento (y valoración) entre diferentes formas de pensar, entre disciplinas y entre países.

\section{Referencias citadas}

ROBERT, J., 2014 - Quintas jornadas de jóvenes americanistas: «Nuevos acercamientos de los desafíos ambientales: las ciencias sociales frente a la adaptación a cambios climáticos. Perspectivas transdisciplinarias, formaciones y diálogos metodológicos». Bulletin de I'Institut Françaos d'Études Andines, 43 (2): 395-402. Reseña de evento.

ORÉ, T. \& DAMONTE, G. (Eds.), 2014 - ¿Escasez de agua? Retos para la gestión de la cuenca del río Ica; Lima: Fondo Editorial PUCP, CISEPA, GIZ. 